Die genannten Beiträge, aber auch die Artikel von Sonja Zmerli und Ken Newton (Winners, Losers and Three Types of Trust) sowie von Tom van der Meer und Paul Dekker (Trustworthy States, Trusting Citizens) bleiben bei bekannten Themen der Vertrauensforschung, bearbeiten diese aber unter neuen Fragestellungen oder auf der Basis neuer Daten und tragen insofern zur Kumulation und Weiterentwicklung empirischer Forschungsbefunde bei. Weniger breit bearbeitete Fragen greifen die Beiträge von Eric Uslaner (Korruption, Ungleichheit und Vertrauen), Staffan Kumlin sowie Eva-Maria Trüdinger und Uwe Bollow auf, wobei letztere die Wechselbeziehungen zwischen wohlfahrtsstaatlicher Politik und politischem Vertrauen behandeln. Während Kumlin das Vertrauen beziehungsweise die Demokratiezufriedenheit als abhängige Variable einführt und sich der Untersuchung des Einflusses makroökonomischer Leistungsparameter auf diese Einstellung widmet, beschäftigen sich Trüdinger und Bollow mit der Bedeutung des Vertrauens als Determinante der Einstellungen zur Reform des Sozialstaates.

Mit dem Band haben die Herausgeber eine Sammlung überwiegend interessanter und lesenswerter Einzelbeiträge vorgelegt, von denen die meisten bekannte Probleme der Vertrauensforschung in neuer Akzentuierung behandeln, während insbesondere die letzten drei bisher wenig bearbeitete Fragen aufgreifen und neue empirische Befunde liefern.

Oscar W. Gabriel

Umbrüche in Italien: Nachschlagewerk und hilfreiche Analyse zu den Grenzen institutioneller Steuerung

Köppl, Stefan: Politik in Italien: Vom Kartell zum Wettbewerb? Parteien - Parlament-Regierung, Nomos Verlagsgesellschaft, Baden-Baden 2011, 387 Seiten, €49,-.

Stefan Köppl beschäftigt sich in seiner Dissertation mit den Umbrüchen im politischen System Italiens in den 1990er Jahren und ihren Auswirkungen auf den politischen Willensbildungs- und Entscheidungsprozess. Er nimmt dabei die 1993 umgesetzten Veränderungen im Wahlrecht als Ausgangspunkt verschiedener Umstrukturierungen im Parteiensystem in den Blick und stellt die berechtigte Frage, ob die ambitionierten Ziele der damaligen Reformer, nämlich die Verbesserung von Repräsentation und Regierbarkeit, tatsächlich erfüllt werden konnten (S. 22 f.).

Der Autor gliedert seine Arbeit nach zwei institutionellen Arenen, die durch die Reformen beeinflusst wurden: die Parteien beziehungsweise das Parteiensystem sowie das Parlament und die Regierung. Er vergleicht diese Arenen in ihrem jeweiligen Zustand vor den Reformen in der Ersten Republik (1946 bis 1991) und nach den Reformen in der Zweiten Republik (1996 bis 2006). Gemäß der Ziele der Wahlrechtsreform berücksichtigt Köppl in beiden Arenen Aspekte, die sich auf die Wahl der Repräsentanten, die Stimmtransformation und das Verhältnis von Wählern und Gewählten beziehen sowie auf den Entscheidungsprozess während der Wahlperiode.

Im ersten Teil der Arbeit untersucht Köppl, inwiefern die Ausrichtung des neuen Wahlrechts an mehrheitsdemokratischen Prinzipien, wie der Mehrheitswahl im Einerwahlkreis, eine Bipolarisierung des Parteiensystems gefördert und der starken Fragmentierung entge- 
gengewirkt hat. Er stellt die Entwicklungen in der Parteienlandschaft vor allem in der Ersten Republik in allen Einzelheiten dar. Dies ist sinnvoll, um das Ausmaß der Fragmentierung nachzuvollziehen. Es besteht allerdings die Gefahr, dass der weniger vorgebildete Leser den Überblick über sämtliche Parteigründungen, -auflösungen und -abspaltungen verliert. Hier hätte auf die Nennung der einen oder anderen Kleinstpartei verzichtet werden können. In der präzisen Analyse wird dann allerdings deutlich, welche Rolle selbst kurzlebige und kleine Parteien in Italien gespielt haben und weiterhin spielen. Ähnlich ist es mit der Wahlrechtsreform: Die Bedeutung der Wahlrechtsänderungen von 1993 („Mattarellum“) und 2005 („Porcellum“) erschließt sich dem Leser in vollem Umfang erst am Ende des ersten Teils. Es wird deutlich, dass es notwendig ist, die Gesetze und vor allem ihre Lücken in allen Einzelheiten zu kennen, um auch die Umgehungsstrategien der Parteien einordnen und bewerten zu können. An dieser Stelle sei nur auf die Beispiele des „scorporo“, einem Kompensationsmechanismus zur Schwächung mehrheitswahl-orientierter Elemente im Wahlrecht, und der „liste civetta“ verwiesen, der Listen mit falschen Kandidaten, deren Zweck es war, ein durch den „scorporo“ hervorgerufenen Stimmabzug abzupuffern und die eigentlichen Listen vor Verlusten zu schützen. Köppl erläutert diese teils absurden Besonderheiten des italienischen Wahlrechts detailliert und dennoch verständlich. Im ersten Teil der Arbeit gelingt es dem Autor damit zu verdeutlichen, wie die Sonderregelungen im Wahlrecht und die Spezifika des italienischen Parteiensystems einen offenen politischen Wettbewerb und das Ideal einer demokratischen und effizienten Politik über Jahrzehnte verhindert haben.

Er kommt hier zu dem Ergebnis, dass sich mit der Bildung der Wahlbündnisse der linken und rechten Mitte vor den Wahlen durchaus eine Bipolarisierung realisieren ließ, dass die starke Fragmentierung sich allerdings weiterhin hält (S. 168 ff.).

Im zweiten Teil nimmt Köppl die parteipolitischen Veränderungen als gegeben an und untersucht ihre Wirkung auf die Arbeit von Parlament und Regierung. Ziel der Reformer war es hier vor allem, eine Erneuerung der Eliten und reale Machtwechsel zu ermöglichen sowie die Bedingungen für ein effizientes und stabiles Regieren zu schaffen. Um den Erfolg dieser Zielsetzungen zu überprüfen, geht Köpplsowohl auf Veränderungen im institutionellen Rahmen als auch auf die politische Praxis ein, die sich unter diesen Bedingungen etabliert hat.

Als Ergebnis fasst er zusammen, dass einzelne Ziele der Reformer - hier ist vor allem der regelmäßige Machtwechsel zu nennen - erreicht werden konnten. Bedingungen für ein verantwortungsvolles Regieren wurden damit prinzipiell geschaffen. Gleichzeitig bestehen die strukturellen Probleme der „Ersten“ Republik in der „Zweiten“ Republik fort: Der politische Alltag wird in Italien mehr durch die Spannungen zwischen Regierung und Parlament bestimmt als durch die klassische Konfliktlinie zwischen Mehrheit und Opposition. Die Koalitionsregierungen, deren heterogene Partner bereits intern für eine hohe Fragilität sorgen, können kaum auf die Unterstützung der Parlamentsmehrheit hoffen; sie sind somit wenig handlungsfähig und der permanenten Gefahr des Vertrauensverlusts ausgesetzt (S. $328 \mathrm{ff}$. .).

Die Gesamtbilanz des Erfolgs der Wahlrechtsreform fällt schließlich gemischt aus: Wenn auch einige Ziele wie eine Bipolarisierung des Parteiensystems erreicht werden konnten, hat sich doch ein Großteil der Strukturen der Ersten Republik halten können (S. 340).

Köppl beantwortet auf hervorragende Art und Weise die brennende Frage, warum die Reformen und Bewegungen der 1990er Jahre das Regieren in Italien nicht effizienter machen 
und damit die italienische Politik im Sinne des Idealtypus der britischen Mehrheitsdemokratie nicht deutlich verbessern konnten. Das zentrale Problem des politischen Systems Italiens hält Köppl sehr pointiert fest: die Koexistenz von sich zum Teil widersprechenden konsens- und mehrheitsdemokratischen Elementen, die für eine massive Irritation der politischen Akteure sorgt und nicht nur ein effizientes, sondern vor allem ein konsequentes Regieren verhindert.

Für den deutschsprachigen Raum ist Köppls Beschreibung und Analyse der historischen und institutionellen Entwicklungen des politischen Systems in Italien hinsichtlich des Umfangs und der Ausführlichkeit einmalig. Seine Literaturgrundlage umfasst sämtliche relevanten italienisch-, deutsch- und englischsprachigen Werke der letzten Jahrzehnte. Damit gewinnt das Buch fast schon den Charakter eines Nachschlagewerks - unter anderem zur Beurteilung der aktuellen politischen Lage Italiens, die durch die massive Staatsverschuldung bestimmt wird. Dass die strukturell bedingten Schwierigkeiten des Regierens Ursache oder zumindest Katalysator für die italienische Schuldenkrise sind, liegt auf der Hand. Der Wert eines solchen Grundlagen schaffenden Werkes wird gerade in derartigen Situationen besonders deutlich.

Sara Braun

\section{Europäische Parteien: umfassender und gut lesbarer Überblick}

\section{Mittag, Jürgen und Janosch Steuwer: Politische Parteien in der EU, UTB: facultas wuv, Wien 2010, 314 Seiten, $€ 18,90$.}

Im Vergleich zum Europäischen Parlament (EP), der Kommission oder dem Rat der Europäischen Union (EU) sind europäische Parteien ein deutlich weniger bearbeitetes Feld. Dies ist letztlich wohl darauf zurückzuführen, dass sie zumindest in der Form grenzüberschreitender Bündnisse nationalstaatlich operierender Parteien (erste Begriffsdimension der Autoren) trotz prominenter Erwähnung in europäischen Verträgen bislang politisch ein Schattendasein führen. Dass ihr Handlungspotenzial durch die Bestimmung ihrer rechtlichen Grundlagen und ihrer finanziellen Ausstattung „deutlich erhöht worden“ (S. 11) ist, wie beide Autoren proklamieren, ist von der Öffentlichkeit fast vollständig unbemerkt geblieben. Schon mehr schon Bedeutung erlangen europäische Parteien durch ihre Fraktionen im Europäischen Parlament (zweite Begriffsdimension); als dritte Begriffsdimension betrachten Jürgen Mittag und Janosch Steuwer die Europäisierungstendenzen nationaler Parteien. Damit legen sie ein weites Verständnis europäischer Parteien zugrunde und bieten gleichzeitig einen umfassenden Überblick über Parteien auf europäischer Ebene und über den Einfluss der europäischen Integration auf Parteien insgesamt. Diese Vielfältigkeit der Perspektive kann als großer Vorteil dieses Buches gelten, selbst wenn sie, worauf weiter unten einzugehen ist, nicht konsequent eingehalten wird. Der Leser erfährt somit viel Wissenswertes über die historischen Entwicklungslinien, den Aufbau und die Funktionen europäischer Parteien, über deren System, ihre Fraktionen im EP und über die Europäisierung nationaler Parteien. Detailliert, anschaulich, faktenreich und graphisch gut aufgearbeitet beschreiben und analysieren die Autoren die einzelnen europäischen Parteienbünde und deren Fraktionen im EP. Sie fassen dabei in jedem Kapitel nicht nur den aktuellen Stand der Parteien- und Parlamen- 